\title{
Specific Heat of the 2D Hubbard Model
}

\author{
Daniel Duffy and Adriana Moreo \\ Department of Physics, National High Magnetic Field Lab and MARTECH, Florida State University, Tallahassee, FL 32306, \\ USA
}

(September 24, 2018)

\begin{abstract}
Quantum Monte Carlo results for the specific heat $c$ of the two dimensional Hubbard model are presented. At half-filling it was observed that $c \sim T^{2}$ at very low temperatures. Two distinct features were also identified: a low temperature peak related to the spin degrees of freedom and a higher temperature broad peak related to the charge degrees of freedom. Away from half-filling the spin induced feature slowly disappears as a function of hole doping while the charge feature moves to lower temperature. A comparison with experimental results for the high temperature cuprates is discussed.
\end{abstract}

PACS numbers: $65.40 .+\mathrm{g}, 65.50 .+\mathrm{m}, 75.40 . \mathrm{Cx}, 75.40 . \mathrm{Mg}$

\section{INTRODUCTION}

The Hubbard model is among the simplest Hamiltonians that describe the behavior of correlated electrons. Specially since the discovery of high temperature superconducting materials, considerable attention has been devoted to this model and significant progress was achieved in understanding its ground state properties, particularly at half-filling, although superconductivity is still elusive [1]. Static and dynamical spin correlations, the optical conductivity and other observables have been studied in detail. [1] However, not much attention has been devoted to its thermodynamical properties despite the large amount of experimental specific heat measurements available for the cuprates. The aim of this paper is to fill that void and to present a systematic study of the specific heat of the two dimensional (2D) Hubbard model for different couplings $U / t$, dopings and temperatures. To achieve that goal Quantum Monte Carlo (QMC) techniques are used.

The Hubbard Hamiltonian is given by

$$
\begin{gathered}
H=-t \sum_{\langle\mathbf{i} \mathbf{j}\rangle, \sigma}\left(c_{\mathbf{i}, \sigma}^{\dagger} c_{\mathbf{j}, \sigma}+\text { h.c. }\right) \\
+U \sum_{\mathbf{i}}\left(n_{\mathbf{i} \uparrow}-1 / 2\right)\left(n_{\mathbf{i} \downarrow}-1 / 2\right)+\mu \sum_{\mathbf{i}, \sigma} n_{\mathbf{i} \sigma},
\end{gathered}
$$

where $c_{\mathbf{i}, \sigma}^{\dagger}$ creates an electron at site $\mathbf{i}$ with spin projection $\sigma, n_{\mathbf{i} \sigma}$ is the number operator, the sum $\langle\mathbf{i j}\rangle$ runs over pairs of nearest neighbor lattice sites, $U$ is the onsite Coulombic repulsion, $t$ the nearest neighbor hopping amplitude, and $\mu$ the chemical potential. In the following $t=1$ will be used as the unit of energy. The boundary conditions are periodic.

\section{HALF-FILLING}

The computational calculation of the specific heat $c$ is not simple. In principle, $c$ is given by the derivative of the energy $E$ (defined as $E=\langle H\rangle / N$, with $N$ being the number of sites) with respect to the temperature $T$ at constant density. However, note that in determinantal QMC simulations, which are set up in the grand canonical ensemble, the energy is a function of the chemical potential that has to be adjusted to keep the density $\langle n\rangle$ constant as the temperature changes. In other words, $\partial E / \partial T$ must be calculated along lines of constant $\langle n\rangle$ in the $T-\mu$ plane. In this framework the calculation of $c$ cannot proceed using $c \sim\left\langle H^{2}\right\rangle-\langle H\rangle^{2}$, as when the number of particles is fixed. Another detail that is important is the finite discretization of the derivatives along the lines of constant density. Naively, the ratio $\Delta E / \Delta T$, with $\Delta T$ very small, should be calculated. However, us-

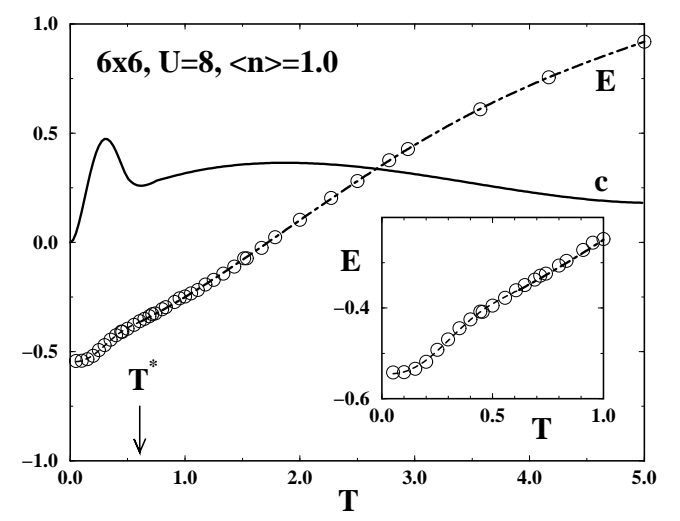

FIG. 1. Monte Carlo results for the energy $E$ on a $6 \times 6$ cluster at half-filling and $U=8$ (open circles). The low temperature polynomial fit is indicated by the dashed line, while the short-long dashed line indicates the high temperature fit. The solid line denotes the specific heat $c$. The low temperature data that produce the spin peak are shown in the inset. The error bars are smaller than the size of the dots. 

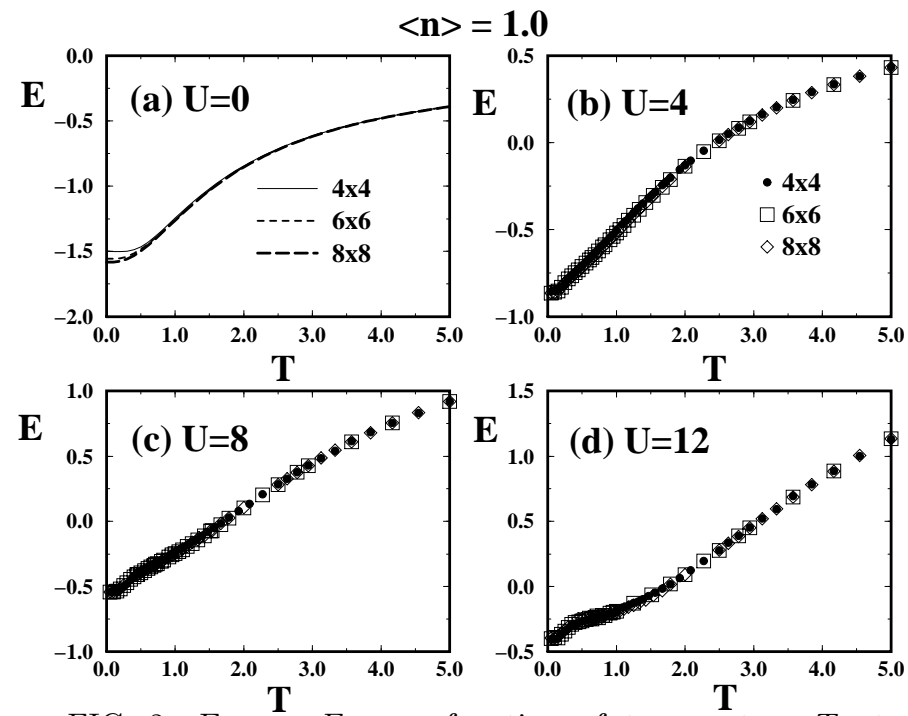

FIG. 2. Energy $E$ as a function of temperature $T$ at half-filling on $4 \times 4,6 \times 6$ and $8 \times 8$ clusters for a) $U=0$, b) $U=4$, c) $U=8$ and d) $U=12$. The error bars are smaller than the size of the dots.

ing such a procedure the small statistical error in $E$ introduces large errors in $c$. For that reason we have decided to calculate $E(T)$ (at fixed $\langle n\rangle$ ) numerically as accurately as possible, and then fit the Monte Carlo points with a polynomial that smears out the small fluctuations in $E$. $c$ is obtained by taking derivatives from this polynomial analytically. Motivated by the shape of the $E$ vs $T$ curve, different polynomials were used for the high and low temperature regimes. In Fig.1, the raw Monte Carlo data for $E$ as a function of temperature corresponding to $U=8$ at half-filling on a $6 \times 6$ cluster are presented. Each data point was obtained by performing around 10,000 measurement sweeps. The dashed line indicates the low temperature fit by a polynomial of order 6 in $T$, while the short-long dashed line indicates the high temperature fit, in this case to a polynomial of order $4 . T^{*}$ is the temp-

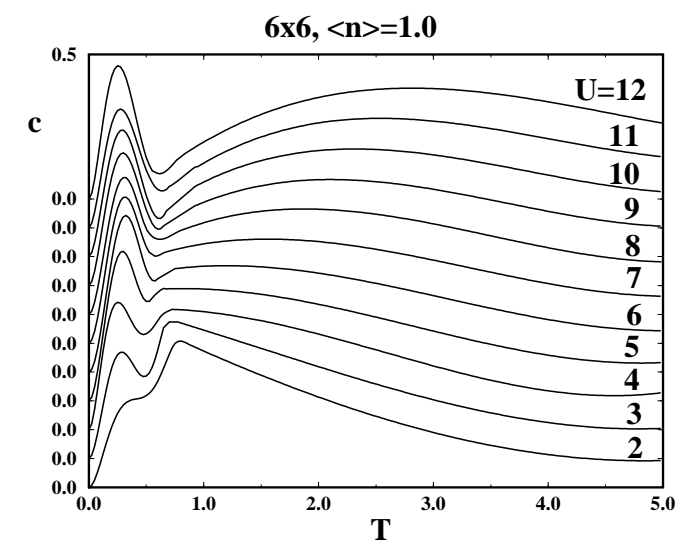

FIG. 3. Specific heat $c$ vs $T$ at half-filling for different values of $U$ ranging from 2 to 12 . The vertical axis for each coupling is shifted for clarity.
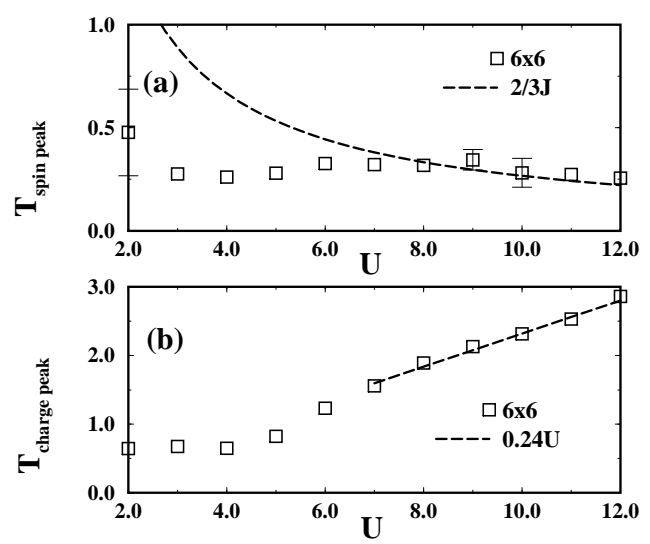

FIG. 4. a) Temperature $T_{\text {spin peak }}$, where the spin peak is located, as a function of $U$ at half-filling. The dashed line indicates $T=2 J / 3$ (asymptotic result in the Heisenberg limit); b) Temperature $T_{\text {charge peak }}$, where the charge peak is located, as a function of $U$. The dashed line indicates $T=0.24 U$.

erature where the two fits meet. Its value depends on the parameters $U$ and $\langle n\rangle$ and is typically of the order of 1 . In order to make a smooth connection of the two fits we included points below (above) $T^{*}$ for the high (low) temperature fit within a window $\sim 0.2$ centered at $T^{*}$. The specific heat was obtained through the analytic derivative of the fitting polynomials, and it is also shown in Fig.1 with a continuous line. The inset of the figure shows with more detail the low energy data that generate the low temperature peak in $c$ (to be discussed later).

An important issue in QMC simulations are finite size effects (FSE). Upon studying $4 \times 4,6 \times 6$ and $8 \times 8$ clusters, it was observed that the FSE in $E$ vs $T$ are strong at very weak coupling but become negligible for $U=8$ or larger. In Fig.2, the energy of the different clusters for $U=0,4,8$ and 12 is shown. Since the FSE are small we decided that results on $6 \times 6$ clusters are representative of the physical behavior analyzed in this study and, thus, this is the lattice size that we have used in the remaining of the paper. In Fig.3, $c$ vs $T$ at half-filling for different values of $U$ is shown. There are two important features in these curves: 1) A low temperature peak that appears when the low lying spin states are excited, and 2) a higher temperature peak which appears when states in the upper Hubbard band are excited. In the weak coupling regime the low temperature peak moves to slightly higher temperature as $U$ increases, reaching a turning point at $U \approx 7$ where the peak is at $T=0.3$. For $U>7$ the peak slowly moves to lower temperatures, as $U$ grows. This indicates the beginning of the strong coupling regime since it is well known that for large values of $U$ the Hubbard and the $t-J$ models have similar behaviors and the coupling constants are related through $J=4 t^{2} / U$. Numerical studies on the $t-J$ model have indicated that at half-filling (Heisenberg limit) the peak in $c$ appears at $T \approx 2 J / 3$ [2] which in terms of $U$ corresponds to $T \approx 8 t^{2} / 3 U$. Thus, when this regime is reached we expect the peak to move to lower temperature with increasing $U$. The position of the peak as a function of 

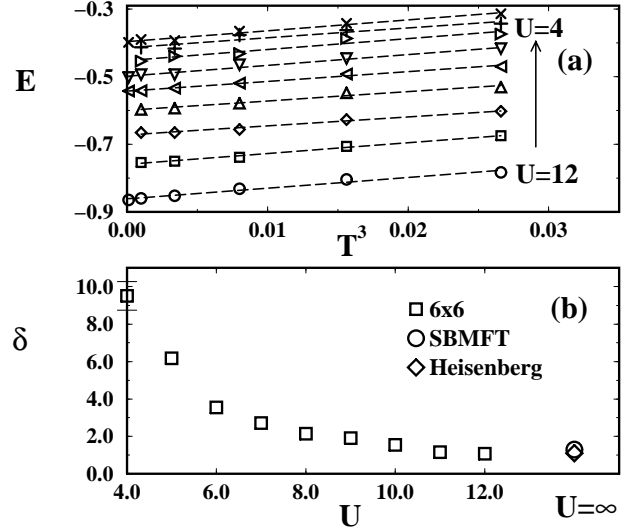

FIG. 5. a) Energy vs $T^{3}$ at half-filling for different values of $U$; b) Coefficient $\delta$ of the low temperature fit $c \sim \delta T^{2}$, as a function of $U$ at half-filling. Numerical and analytical values for the Heisenberg limit $(U=\infty)$ are indicated. The circle corresponds to the mean field result of Ref.[6], and the diamond denotes the numerical result of Ref.[8].

$U / t$ is shown in Fig.4.a, where the dashed line indicates $T=2 J / 3$. The asymptotic behavior is reached for $U \geq$ 10 .

The broad high temperature peak moves to higher temperature as $U$ increases as expected since its presence corresponds to the excitation of states across the gap that grows with $U$. In Fig.4.b the position of this peak is shown as a function of $U$. For $U \geq 7$ the dependence of the position of the peak with $U$ becomes approximately linear, and it is given by $0.24 U$. A spin-density-wave mean field calculation of the gap $\Delta$ as a function of $U$, at large $U$, gives the result $\Delta \sim 0.48 U$. Apparently, quantum fluctuations reduce the size of the gap. Note that in Fig. 3 it can be observed that the minimum in $c$ between the two peaks becomes deeper as $U$ increases and the charge peak increases its width.

In previous work the specific heat for the half-filled Hubbard model in one dimension has been evaluated. [3,4.5] We found that the qualitative behavior in two and one dimensions is similar regarding the existence and coupling dependence of the two peaks. However, the following differences were observed: 1) According to Ref. [3] the two peaks can be resolved for $U>4$ while here we were able to identify the two peaks already at $U=2$. The fact that only one maximum is observed in Ref. [3] in the strong coupling regime is due to the small $T$ interval considered in their study; 2) According to Ref. (4) the maximum in $c$ associated with the spin excitations moves to lower temperatures as $U$ increases in weak coupling while in our 2D study the opposite behavior was found.

Another important feature observed here at half-filling is that at low temperatures the specific heat follows $c=\delta T^{2}$, i.e., the behavior predicted by spin-wave calculations. [6] In Fig.5.a we show the energy as a function of $T^{3}$ for different values of $U$ showing that linear behavior occurs for $T \leq 0.3$. The value of $\delta$ depends on $U$, and it decreases as the coupling increases. For large $U$ the

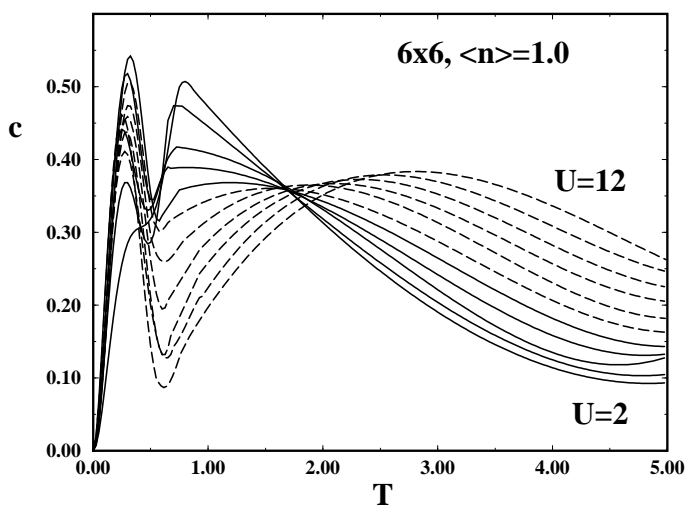

FIG. 6. $c$ vs $T$ for $U=2$ to 5 (continuous lines) and for $U=6$ to 12 (dashed lines). All the curves intersect at $T_{2} \approx 1.6$, while those corresponding to weak coupling (continuous lines) also intersect at $T_{1} \approx 0.6$.

limiting value $\delta \approx 1.1$ is obtained in good agreement with the reported value for the Heisenberg model. [6, 6], 8 A slave boson mean field theory (SBMFT) calculation provided a value of $\delta=1.3 \pm 0.05$ [6] while a numerical study obtained $\delta=1.1 \pm 0.2$. [8] The behavior of $\delta$ vs $U$ is shown in Fig.5.b.

In Fig.6, $c$ vs $T$ for several values of $U$ ranging from 2 to 12 are presented. These are the same curves that were shown in Fig.3 but now using common vertical units. It is interesting to observe that all the curves intersect at $T=1.6 \pm 0.2$. If only small values of the coupling are considered, i.e. $U$ ranging from 2 to 5 , the curves cross also at $T_{1}=0.6 \mathrm{in}$ addition to $T_{2}=1.6$. This behavior was predicted by Vollhardt $[9]$ and was observed in the paramagnetic phase of the infinite dimensional Hubbard model for $0 \leq U \leq 2.5$.

\section{FINITE HOLE DENSITY}

To compare our results with those of the superconducting cuprates it is important to study the specific heat as
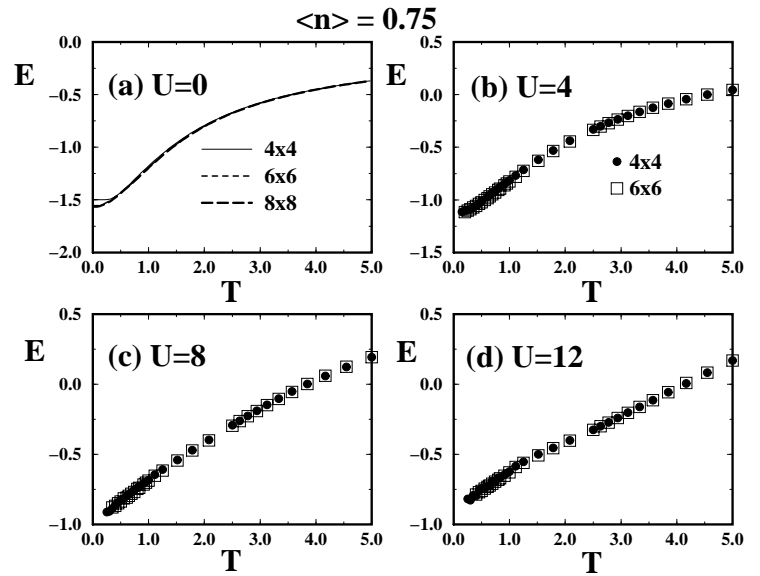

FIG. 7. Energy $E$ as a function of temperature $T$ on $4 \times 4$ and $6 \times 6$ clusters and density $\langle n\rangle=0.75$ for a) $U=0$, b) $U=4$, c) $U=8$ and d) $U=12$. 


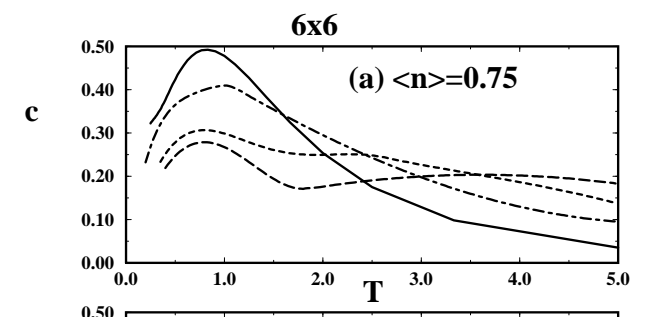

c

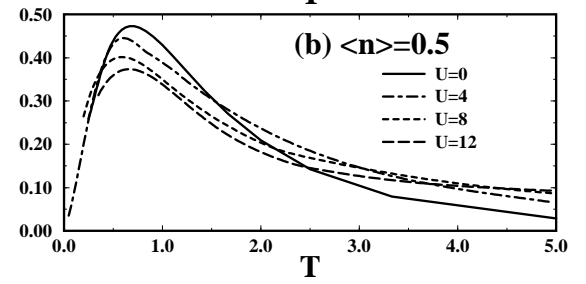

FIG. 8. $c$ vs $T$ at density a) $\langle n\rangle=0.75$ and b) $\langle n\rangle=0.50$ for $U=0,4,8$ and 12 , on a $6 \times 6$ cluster.

a function of hole doping. As remarked before, many experimental measurements of the specific heat for high temperature cuprates are available. In general it is very difficult to separate the electronic contribution to the specific heat in the normal state from the phononic part. Also many experiments have been performed in the superconducting phase, where the existence of an intrinsic linear contribution to the specific heat would indicate the absence of a gap and thus non-conventional behavior. 10,11,12 Since the superconducting phase cannot be reached in QMC simulations, our results will be compared with experiments performed in the normal state. For $\mathrm{La}_{2-x} \mathrm{Sr}_{x} \mathrm{CuO}_{4}$ it was observed in Ref. 13] that the linear term $\gamma$ of the specific heat in the normal phase increases with doping between $x=0.12$ and 0.25 . However, studies of the same material performed later 14 showed that $\gamma$ increases with $x$ for $x>0.1$ reaching a maximum value at optimal doping $x \approx 0.15$ and then decreasing in the overdoped regime. This behavior is in agreement with the Van Hove scenario [15] where the density of states reaches a maximum at optimal doping. The behavior of $\gamma$ for a metal-insulator transition was also studied for $\mathrm{Sr}_{1-x} \mathrm{La}_{x} \mathrm{TiO}_{3}$ in Ref. [16]. They observed that $\gamma$ increases as the transition is approached from the metallic side. Through the relation $\gamma=m^{*} \gamma_{0} / m$, where $\gamma_{0}$ and $m$ are the linear coefficient and the mass for free electrons, it was found that the effective mass of the quasiparticles $m^{*}$ increases as the transition is approached. Loram et al. 177 studied $\gamma$ as a function of doping at $T=280 \mathrm{~K}$ in $Y B a_{2} C u_{3} O_{6+x}$. $\gamma$ appears to increase with doping reaching a plateau for $x \approx 0.45$.

The first step to study numerically the specific heat at finite density is to analyze the finite size effects. They are stronger than at half-filling, but still moderate as can be seen in Fig.7 where $E$ vs $T$ for $U=0,4,8$ and 12 at $\langle n\rangle=0.75$ on $4 \times 4$ and $6 \times 6$ clusters is shown. Away from half-filling it was very difficult to obtain accurate results on $8 \times 8$ clusters at low temperature due to the wellknown sign problem. However, since FSE are stronger in weak coupling and we have observed that for $U=0$, where results can be obtained exactly, there is only a

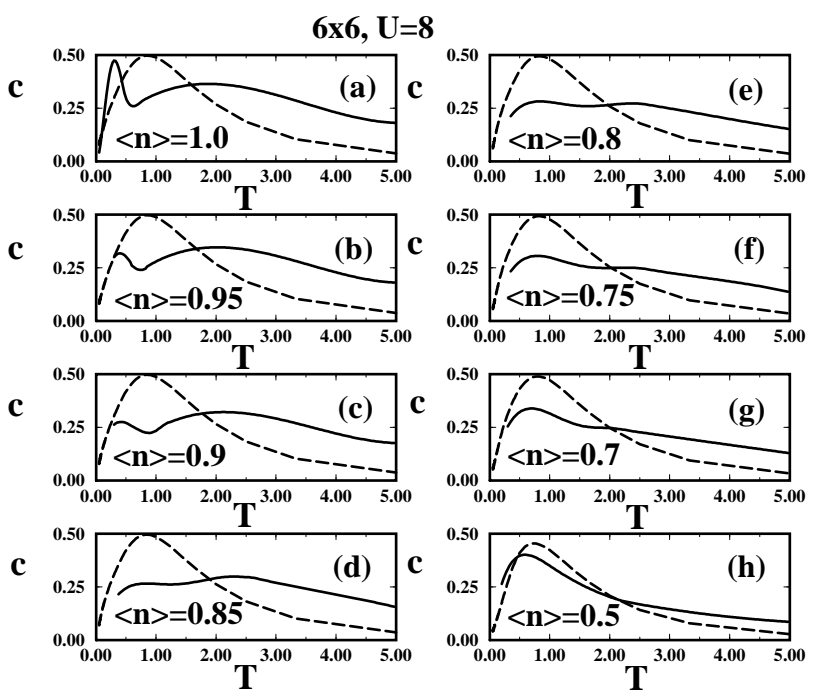

FIG. 9. $c$ vs $T$ for $U=8$ (solid line) on a $6 \times 6$ cluster at different densities. The dashed line indicates results for $U=0$ obtained using a $200 \times 200$ cluster.

small difference between the $6 \times 6$ and $8 \times 8$ results, then as before, $6 \times 6$ lattices were used in our studies away from half-filling.

In Fig.8.a the specific heat as a function of $T$ at $\langle n\rangle=0.75$ for different values of $U$ is presented. It can be observed that the spin peak is substantially reduced compared with the results at half-filling, but it is still present in strong coupling for $U=8$ and 12 indicating the existence of short range antiferromagnetic correlations. In weak coupling, i.e. for $U=4$, the spin feature has disappeared, and the curve is similar to the non-interacting one. The specific heat increases in the region where the minimum between the two peaks existed at half-filling. At quarter filling (Fig.8.b), $c$ has a behavior that resembles free electrons independently of the value of $U$. Thus, here the electrons are approximately weakly interacting at all couplings.

Let us consider in more detail the special case of $U=8$. This value of the coupling was selected since according to calculations of the optical conductivity it is suitable to reproduce some normal state experimental results [1]. In Fig.9 the specific heat as a function of temperature is presented for different values of the density $\langle n\rangle$. The continuous line indicates the results for $U=8$ on a $6 \times 6$ cluster while the dashed line denotes the non-interacting $U=0$ results on a $200 \times 200$ lattice. Such a large cluster in the non-interacting case was used to avoid finite size effects which are strong in this limit at the low temperatures where the linear behavior occurs. Again it should be remarked that this problem occurs in weak coupling at very low $T$ and, thus, our $U=8$ results are not expected to be contaminated by size effects. In Fig.9 it can be seen that for $U=8$ the intensity of the spin peak decreases smoothly with doping. At $10 \%$ hole doping (i.e. $\langle n\rangle=0.90)$ its intensity diminishes by $40 \%$, a result in agreement with Ref. [2] where the $t-J$ model was studied. Note that for $\langle n\rangle \sim 0.8$ the specific heat is almost 


\section{$6 \times 6, U=8$}
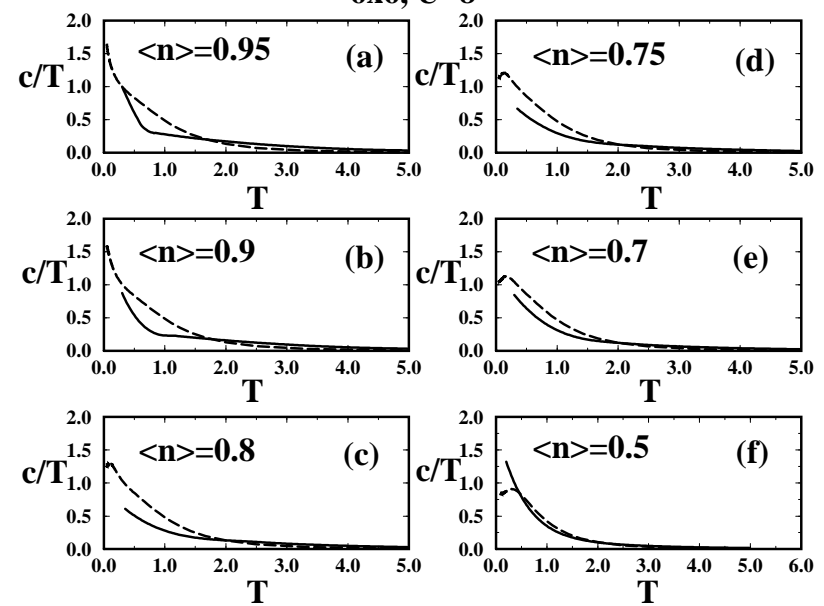

FIG. 10. $c / T$ vs $T$ for $U=8$ (solid line) on a $6 \times 6$ cluster at different densities. The dashed lines denote results for $U=0$ obtained using a $200 \times 200$ cluster.

flat in a broad range of temperatures. Here it is difficult to resolve the spin and charge peaks from the data. We expect that at this density or lower the spin correlations are no longer important, even those of short-range, in agreement with previous spectral function studies performed in the Hubbard model. [18] Reducing further the density from $\langle n\rangle=0.75$ to 0.5 a single peak structure that resembles the non-interacting specific heat curve becomes dominant.

An important issue in this context is the calculation of couplings and densities where the system changes from insulator to metal. Metallic behavior is characterized in the specific heat by the existence of a linear coefficient $\gamma$. In two dimensions it was found that [19]

$$
c \approx \gamma T+\Gamma_{2 D} T^{2}+\ldots,
$$

with $\Gamma_{2 D}$ positive in strong coupling.

The experimentalists often present plots of $c / T$ vs $T^{2}$ when addressing $\gamma$. Analogously, in Fig.10 the continuous line denotes $c / T$ vs $T$ for $U=8$ at different densities, while the dashed line indicates the non-interacting case. The lowest temperature that was confidently reached in this study away from half-filling is $T=0.3$. It is clear that this temperature is too high to observe the linear behavior in $c / T$ since, according to Eq.(2), the slope of the curve has to be positive at very low temperature. Clearly, if the system behaves as a Fermi liquid a maximum has to appear in the curve at a lower temperature than reached in this study. The non-interacting results show indeed the linear behavior at very low temperatures. However, note that the value of $\gamma$ for non-interacting electrons is not much different from the value of $c / T$ at the maximum in Fig.10 at all densities. Thus by extrapolating the $U=8$ curves to zero we expect to obtain a good

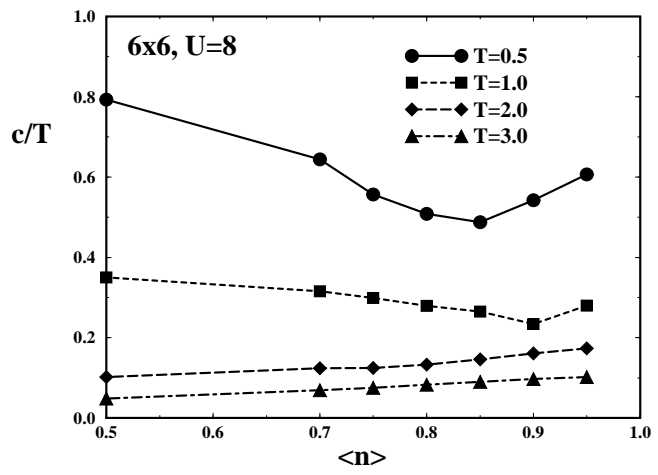

FIG. 11. $c / T$ vs $\langle n\rangle$ for $U=8$ at different temperatures.

approximation to the value of $\gamma$. However, since we cannot reach lower temperatures the existence of anomalous non-Fermi liquid behavior can certainly not be ruled out as remarked in Ref. [2].

In Fig.11, $c / T$ as a function of doping is presented at different temperatures. Notice that the lowest temperature $T=0.5$ shown in Fig. 11 corresponds to $\sim 2000 K$, if $t=0.4 \mathrm{eV}$ is used. This is much higher than $T=280 \mathrm{~K}$ which is the highest temperature used in experiments. However, for $T=0.5$ it was here observed that $c / T$ increases with doping for $\langle n\rangle \leq 0.8$ in agreement with some experimental results [17], and the same behavior is observed at $T=1$. For higher $T$ the ratio $c / T$ increases for increasing density $\langle n\rangle$.

\section{SUMMARY}

The specific heat of the two dimensional Hubbard model has been calculated for different couplings and electronic densities as a function of temperature. At half-filling and as the coupling $U$ increases a low temperature peak associated with the spin degrees of freedom moves to lower temperatures, while a high temperature feature associated with the charge degrees of freedom moves to higher temperatures. At very low temperatures $c \approx \delta T^{2}$ as predicted by spin-wave theory and $\delta$ tends to the Heisenberg value $(\delta \approx 1.1)$ for large coupling $U$. Away from half-filling we observed that the spin feature weakens with doping, and it disappears for $\langle n\rangle \leq 0.75$ working at $U=8$. This suggests the absence of important antiferromagnetic correlations below that density. We were not able to reach temperatures low enough to decide whether the system is metallic or has anomalous behavior away from half-filling. However, by evaluating $c / T$ we were able to make comparisons with experimental results. At the lowest temperatures that we could reach we found that $c / T$ increases with hole doping for $\langle n\rangle<0.9$. This behavior is similar to experimental results for $\mathrm{YBa}_{2} \mathrm{Cu}_{3} \mathrm{O}_{6+x}$. 17 


\section{ACKNOWLEDGEMENTS}

We thank E. Miranda, K. Bedell, S. von Molnar and E. Dagotto for useful conversations. A.M. is supported by NSF under grant DMR-95-20776. Additional support is provided by the Office of Naval Research under grant N00014-93-0495, the National High Magnetic Field Lab and MARTECH. We thank ONR for providing access to their Cray-YMP and CM5 supercomputers.
[1] E. Dagotto, Rev. Mod. Phys. 66, 763 (1994).

[2] J. Jaklic and P. Prelovsek, preprint.

[3] J. Schulte and M. Böhm, Phys. Rev. B 53, 15385 (1996).

[4] T. Usuki, N. Kawakami and A. Okiji, J. Phys. Soc. Jap. 59, 1357 (1990).

[5] A. Klümper and R.Z. Bariev, Nucl. Phys. B458 [FS], 623 (1996).

[6] A. Auerbach and D. Arovas, Phys. Rev. Lett. 61, 617 (1988).

[7] T. Barnes, J. Mod. Phys. C2, 659 (1991).

[8] Y. Okabe, M. Kikuchi and A.D.S. Nagi, Phys. Rev. Lett. 61, 2971 (1988).

[9] D. Vollhardt, preprint.

[10] S.E. Stupp and D.M. Ginsberg, Physica C158, 299 (1989).

[11] K. Mori et al., Physica B165\&166, 1201 (1990).

[12] S.J. Collocott et al., Physica B165\&166, 1329 (1990).

[13] J.W. Loram et al., Physica C162-164, 498 (1989).

[14] N. Wada et al., Physica B165\&166, 1341 (1990).

[15] R.S. Markiewicz, J. Phys. Condens. Matter 2, 6223 (1990); D.M. Newns, P.C. Pattnaik and C.C. Tsuei, Phys. Rev. B 43, 3075 (1991); E. Dagotto, A. Nazarenko and A. Moreo, Phys. Rev. Lett. 74, 310 (1995).

[16] Y. Tokura et al., Phys. Rev. Lett. 70, 2126 (1993); K. Kumagai et al., Phys. Rev. B 48, 7636 (1993).

[17] J.W. Loram et al., Phys. Rev. Lett. 71, 1740 (1993).

[18] S. Haas, A. Moreo and E. Dagotto, Phys. Rev. Lett. 74, 4281 (1995); A. Moreo et al. Phys. Rev. B 51, 12045 (1995).

[19] D. Coffey and K. Bedell, Phys. Rev. Lett. 71, 1043 (1993). 